\title{
The Genetics Corner: A Preterm Infant with Down Syndrome Complicated by Severe Transient Abnormal Myelopoiesis
}

Robin Dawn Clark, MD

\section{Clinical Summary}

A genetics consultation was requested for an 18 day old 30-week two-day gestation male with prenatally diagnosed tetralogy of Fallot and an increased risk for Trisomy 21 by quad screen and NIPT. He had an elevated WBC of 107 at birth with a presumed transient leukemoid reaction and subsequent brain hemorrhage, acute kidney injury, coagulopathy, cholestasis, transaminitis, and non-occlusive portal vein thrombus.

\section{"He had an elevated WBC of 107}

at birth with a presumed transient

leukemoid reaction and subsequent

brain hemorrhage, acute kidney injury, coagulopathy, cholestasis, transaminitis, and non-occlusive portal vein thrombus."

He was born to a 39-year old G3P1SAb1 mother who was offered and declined genetic counseling for advanced maternal age and after a diagnosis of the fetal cardiac defect and a positive NIPT screening test for Down syndrome. An amniocentesis was not performed. The mother presented in preterm labor that did not respond to tocolysis (Magnesium). The male infant was delivered by primary $\mathrm{C}$-section for breech presentation and preterm labor. Apgar scores were 3, 6, and 8 at 1, 5, and 10 minutes.

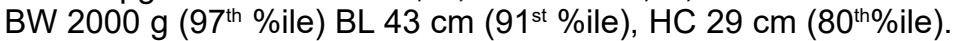
The initial venous $\mathrm{pH}$ was 7.12, $\mathrm{pCO} 271, \mathrm{BE}-7.3$. The initial CBC showed an elevated WBC of 107.45 and Platelets 360,000 and $38 \%$ blasts. He has required multiple transfusions for anemia and thrombocytopenia. His current WBC was 12.74, RBC 2.7, Hgb 8.7, Hct 24.3, and Platelets 41. He had direct hyperbilirubinemia and worsening transaminitis. He had acute kidney injury and was in the polyuric phase, on diuretics. Chromosome analysis was initially unsuccessful due to a lack of metaphases despite stimulation with pokeweed mitogen (B cell mitogen). Repeat chromosome analysis confirmed Trisomy 21 . GATA1 gene analysis was pending.

A recent abdominal ultrasound showed a non-occlusive thrombus of the left portal vein (noted initially on day 3 of life), slow flow in the IVC and main portal vein, and pulsatile flow in the portal vein. There was reversed flow in the right portal vein and likely in the left portal vein. At about two weeks of age, a head ultrasound exam showed bilateral hemorrhages (Grade 3/4) with worsening ventriculomegaly. The most recent echocardiogram showed tetralogy of Fallot, PDA, moderate tricuspid valve regurgitation, and moderate elevation of pulmonary pressures.

On physical examination, the infant was orally intubated, mechanically ventilated, and sedated with morphine. He had anasarca and features of Down syndrome. The abdomen was distended, tense, and tender with guarding. There was a prominent venous pattern on the abdomen, superior to the umbilicus. The liver edge was palpable $5 \mathrm{~cm}$ below the $R$ costal margin. A spleen tip was palpable. The baby had generalized jaundice with a bronze skin tone and scattered petechiae on the torso and extremities.

\section{Discussion}

The question posed to the genetics service was whether this baby with presumed Down syndrome had a second disorder responsible for his complicated course and, if so, whether further genetic testing was warranted. Were his features attributable to transient abnormal myelopoiesis (TAM)? A literature review confirmed that the pattern of multiorgan failure, edema, pulmonary hypertension, coagulopathy, anemia, hepatic dysfunction and possible fibrosis, and cholestasis in this patient have all been reported in severe cases of TAM. No further genetic testing was recommended.

\section{"TAM, also called transient leukemia or transient myeloproliferative disorder, is a clonal expansion of immature megakaryocytes (blasts) in $5-10 \%$ of infants with Down syndrome, which is likely to be of fetal onset."}

TAM, also called transient leukemia or transient myeloproliferative disorder, is a clonal expansion of immature megakaryocytes (blasts) in $5-10 \%$ of infants with Down syndrome, which is likely to be of fetal onset. There is evidence that TAM blasts derive from fetal hepatic hematopoiesis rather than bone marrow. Although there are no clear diagnostic criteria for TAM, a clonal GATA1 (GATA binding protein 1) mutation with or without blasts in the peripheral smear confirms the diagnosis of TAM. In most cases, TAM is asymptomatic, self-limiting, and requires no treatment; spontaneous regression usually occurs within the first few months of life. However, newborns with TAM can present with organomegaly, pericardial effusion, and coagulation disorders. In about $20 \%$ of patients with TAM, serious and life-threatening sequelae develop, including hydrops fetalis, anasarca, direct hyperbilirubinemia, hepatic fibrosis/failure, disseminated intravascular coagulation, and death. In its most severe form, TAM is diagnosed in hydropic stillborn infants with Down syndrome.

TAM is a preleukemic disorder. In an excellent review on the subject, Watanabe (2019) notes that the circulating blast in TAM

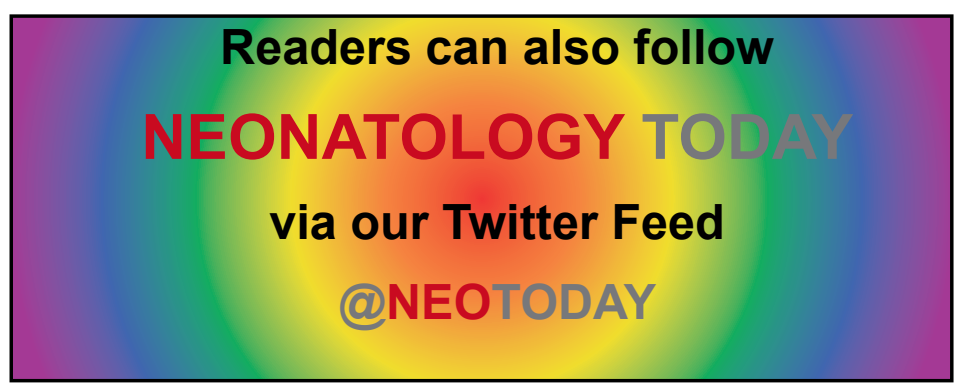


shares morphologic and phenotypic traits similar to blasts from acute myeloid leukemia associated with Down syndrome (MLDS). Among survivors of TAM, about $20 \%$ go on to develop MLDS within the first years of life. Children with no clear history of TAM may also develop ML-DS. These children may have a subclinical clonal disease called "silent TAM" that is detectable only with GATA1 gene analysis utilizing highly sensitive next-generation sequencing techniques.

Dorman and colleagues (2004) reviewed life-threatening complications in five consecutive neonates with Down syndrome and TAM, three of whom were preterm. Four infants, who were critically ill, were treated with cytosine-arabinoside (Ara-C, cytarabine). All five infants survived. Their complications included pulmonary hypertension [ $n=1]$, congestive heart disease [n=2], respiratory failure [n=4], pericardial effusion [n=3], abnormal coagulation studies, renal insufficiency, hepatosplenomegaly, hyperbilirubinemia, hydrops fetalis [n=2], and elevated liver enzymes [ $n=3]$. One female, born at 36 weeks four days gestation, who was treated with Ara-C, required RBC and platelet transfusions for nine weeks before her blood counts normalized. Another female who was treated with Ara-C went on to develop ML-DS at age 20 months.

Flasinski et al. (2018) demonstrated that low-dose cytarabine treatment helped to reduce TAM-related mortality but did not alter the risk for ML-DS. These authors used low-dose cytarabine to treat German and Dutch patients with Down syndrome who had symptomatic TAM or minimal residual disease (MRD). In their cohort of 102 patients with TAM, all patients had either greater than $5 \%$ myeloid blasts or a GATA1 variant detected in exons $1-3$. Of the 81 patients who were molecularly characterized, 78 $(96 \%)$ showed a mutation in GATA1. Eight-six patients entered the intention-to-treat phase of the study. Of these, treatment with low dose cytarabine was completed per protocol in 28/43 symptomatic patients with TAM (high WBC [n=17], hepatic dysfunction and/or cholestasis [ $n=30]$, hydrops fetalis [n=7]), and 11/14 asymptomatic patients with MRD at eight weeks, as determined by flow cytometry. No treatment was offered to an asymptomatic and MRD negative group of 29 patients. Although low dose cytarabine treatment did not change the rate of progression from TAM to ML-DS, the authors observed a significantly lower cumulative incidence of early death in the treated symptomatic TAM group [ $n=28]$ compared to historical controls [ $n=45]$ : $11+/-6 \%$ vs. $33+/-$ $7 \%\left(P_{\text {Gray }}=0.03\right)$.

Yamato and coauthors (2021) performed a meta-analysis to identify risk factors that predict leukemia in patients with TAM. They found that no clinical parameters predicted leukemia development. However, in the subgroup of 36 patients with high WBS count $\left(>100 \times 10^{9} / L\right)$, low dose cytarabine therapy significantly improved survival $(P=0.017)$. They concluded that TAM patients with life-threatening symptoms should be treated with low-dose cytarabine to reduce the early death rate, and flow cytometric MRD positivity at three months was a significant predictive indicator for leukemia development in patients with TAM.

Although there are many unanswered questions about TAM and ML-DS, our evolving understanding of their interconnected pathogenesis raises the hope that improved diagnosis and management of TAM will result in a longer and healthier life for children with Down syndrome.

\section{Practical Applications}

1. Transient abnormal myelopoiesis (TAM) is not always a benign self-limited disease. TAM is a serious and life-threatening disease in up to $20 \%$ of affected infants. Complications such as hydrops fetalis, pulmonary hypertension, anemia, coagulopathy, and hepatic fibrosis contribute to significant TAM-related mortality.

2. Low-dose cytarabine is an effective therapy that reduces mortality in severe TAM.

3. Order a CBC with a differential to check for blasts in all newborns with Down syndrome to detect asymptomatic "silent" TAM.

4. TAM survivors, even those with "silent" TAM, are at increased risk for myeloid leukemia associated with Down syndrome and should be monitored closely.

\section{References}

1. Dormann S, Krüger M, Hentschel $R$, et al. Life-threatening complications of transient abnormal myelopoiesis in neonates with Down syndrome. Eur J Pediatr. 2004 Jul;163(7):3747. Erratum in: Eur J Pediatr. 2005 May;164(5):335. PMID: 15138809

2. Flasinski M, Scheibke K, Zimmermann M, et al. Low-dose cytarabine to prevent myeloid leukemia in children with Down syndrome: TMD Prevention 2007 study. Blood Adv. 2018 Jul 10;2(13):1532-1540. PMID: 29959152

3. Watanabe K. Recent advances in the understanding of transient abnormal myelopoiesis in Down syndrome. Pediatr Int. 2019 Mar;61(3):222-229. PMID: 30593694

5. Yamato G, Deguchi T, Terui K, et al. Predictive factors for the development of leukemia in patients with transient abnormal myelopoiesis and Down syndrome. Leukemia. 2021 May;35(5):1480-1484. PMID: 33654203

Disclosures: The authors have no relevant disclosures.

NT

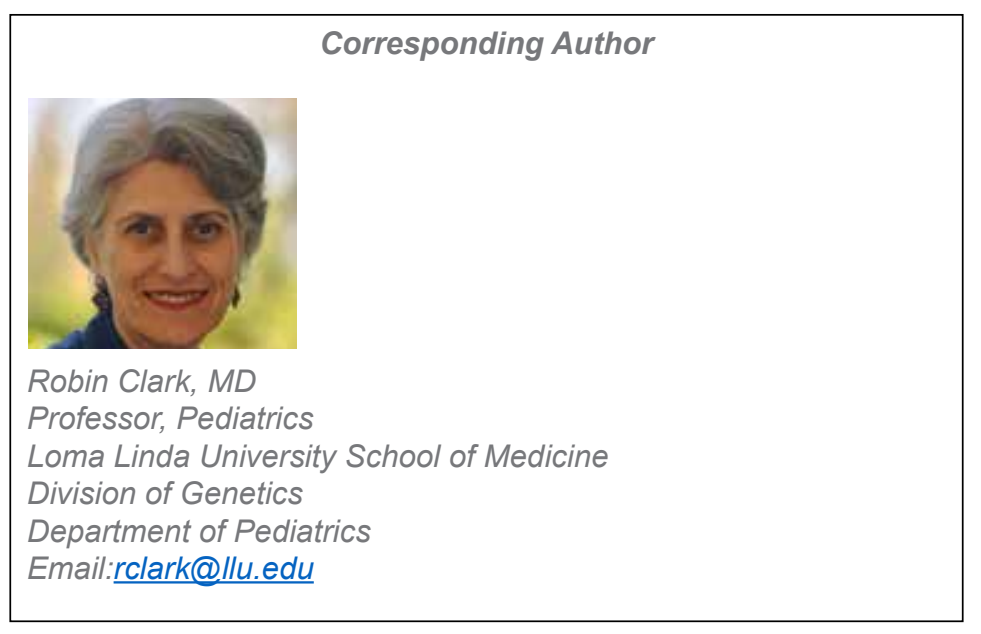

The only worldwide monthly publication
exclusively serving Pediatric and Adult
Cardiologists that focus on Congenital/
$\begin{aligned} & \text { Structural Heart Disease (CHD), and } \\ & \text { CARGENITAL } \\ & \text { Cardiogr }\end{aligned}$

